\title{
Optimizing treatment with thioguanine derivatives in inflammatory bowel disease
}

\author{
Edouard Louis MD, $\mathrm{PhD}$ Jacques Belaiche MD, $\mathrm{PhD}$ \\ Service de Gastroentérologie, CHU de Liège, 4000 Liège, Belgium
}

\begin{abstract}
Thioguanine derivatives, azathioprine and 6-mercaptopurine, represent major drugs in the treatment of chronic active inflammatory bowel disease. They are effective in two-thirds of the patients and safe over the long term in patients who can tolerate them (80-90\%). Recent progress in understanding the metabolism of these drugs and its implication in clinical practice have brought up new tools and strategies that are proposed to optimize treatment. In particular, the measurement and characterization of key enzymes and metabolites may have clinical impact. Thus, thiopurine methyl transferase genotyping and activity measurement, as well as erythrocytes, 6-thioguanine nucleotides and 6-methyl mercaptopurine levels, may help in some situations of intolerance or inefficacy with these drugs. Indications for starting and stopping treatment with thioguanine derivatives are also discussed.
\end{abstract}

Keywords : Crohn's disease ; ulcerative colitis ; inflammatory bowel disease ; azathioprine ; 6-mercaptopurine ; 6-thioguanine nucleotides ; thiopurine methyl transferase.

Thioguanine derivatives, essentially azathioprine (AZA) and 6-mercaptopurine (6-MP), have been used for decades in the treatment of inflammatory bowel disease (IBD). They are considered to be major drugs in the treatment of steroid-dependent and chronic active IBD. A meta-analysis clearly showed that their efficacy in Crohn's disease (CD) correlates with the cumulative dose administered, depending on the dose and duration of treatment. ${ }^{1}$ At a dosage of 1.0-1.5 mg/kg of 6-MP and 2.0-2.5 mg/kg of AZA, one can expect two-thirds to threequarters of the patients to respond after 3-4 months of treatment. ${ }^{2-5}$ More recently AZA and 6-MP have also proved to be useful as maintenance treatment in patients treated with infliximab, probably increasing the response rate ${ }^{6}$ and diminishing the risk of infusion reactions. ${ }^{7}$ Fewer data have been published in ulcerative colitis (UC), but available controlled data ${ }^{8-10}$, as well as reference centre experience ${ }^{5,11}$ indicate similar efficacy. Tolerance and safety over the short and the long term have also been shown to be reasonably good. Two types of adverse reactions can occur in $15-25 \%$ of the patients ${ }^{5,12-14}$ : allergic type and non-allergic type.

The first type usually occurs within the first 3-4 weeks of treatment and is not dose-dependent. It includes pancreatitis (3\%), fever, rash, malaise (2\%), digestive intolerance (10\%) and some cases of hepatitis $(0.3-3 \%)$. The second type usually occurs later, at any time, is dose-dependent and includes bone marrow depression (2$5 \%$ ), infections (2.5-7.5\%), malignancies (3-5\%) and some cases of hepatitis. Importantly, the relative risk of malignancy does not seem to be increased even with long-term treatment. ${ }^{14}$ An association of good efficacy and good tolerance may translate into improved quality of life. This has been shown with AZA/6-MP in inflammatory bowel disease. ${ }^{15}$ In this study, the immunosuppressive treatment (which was mainly AZA) was the main factor improving quality of life.

Despite this rather good profile, improvement can always be sought. Optimize treatment with these drugs includes better defining indication and place in therapeutic strategies of IBD, accelerating and increasing efficacy, improving tolerance and safety. To achieve these aims, our understanding of the metabolism, biochemistry and pharmacogenetics of thioguanine derivatives must be improved. Thanks to recent progress in these fields, several tools are now available and new strategies are proposed, which we are going to develop. For practical and didactic reasons, these will be developed in an order corresponding to the phases of treatment, starting with the initiation of treatment, the early phase, mid- and long-term treatment, and finally the decision to stop treatment. For these periods, available tools and proposed strategies will be discussed, referring when possible to the metabolism of these drugs.

\section{METABOLISM OF THIOGUANINE DERIVATIVES (Figure I)}

Among thioguanine derivatives, three compounds can be used for the treatment of IBD: AZA (Figure I, no. I) 
and 6-MP (Figure I, no. 2), which are the most commonly used drugs, and also 6-thioguanine (6-TG) (Figure I, no. 3), which is used mainly by haematologists in acute lymphoblastic leukaemia in children. Actually, AZA is quickly transformed into 6-MP by a non-enzymatic reaction using glutathione (Figure I, no. 4) or other sulfhydryl-containing proteins with the production of a nitro-imidazole derivative (Figure I, no. 13) that may have some immunomodulatory properties. ${ }^{16}$ 6-MP (Figure I, no. 2) is then itself metabolized through three competitive enzymatic pathways mediated by xanthine oxidase (Figure I, no. 7), thiopurinemethyltransferase (TPMT) (Figure I, no. 5) or hypoxanthine guanine phosphoribosyl transferase (HGPRT) (Figure I, no. 6). The pathway of xanthine oxidase (XO) (Figure I, no. 7) leads to a presumed inactive metabolite, 6-thiouric acid (6TUA) (Figure I, no. 14), and can be inhibited by allopurinol (Figure I, no. 17). The XO gene does not seem to be polymorphic but $2 \%$ of the general population may have a deficient gene giving rise to hypouricaemia. The pathway of TPMT (Figure I, no. 5) leads to 6-methylmercaptopurine (6-MMP) (Figure I, no. 15). The TPMT gene is polymorphic in the general population, with high- and low-metabolizing alleles, generating three possibilities for the rate of metabolism of 6-MP: two high-metabolizing alleles inducing a high-metabolizing pathway ( $88.6 \%$ of the population), one high- and one low-metabolizing allele, inducing an intermediatemetabolizing pathway (11.1\% of the population) and two low-metabolizing alleles, inducing a low-metabolizing pathway $\left(0.3 \%\right.$ of the population). ${ }^{17}$ TPMT activity can also be influenced by exogenous factors, including other drugs, such as diuretics, and AZA itself, that can induce TPMT activity (Figure I, no. 18) while sulfasalazine and mesalamine may have an inhibitory effect (Figure I, no. 19). ${ }^{\mathrm{I}}$ 6-MMP (Figure I, no. 15) is thought to be inactive but seems to mediate some side-effects, including hepatotoxicity. ${ }^{19,20}$ The HGPRT pathway (Figure I, no. 6) leads to 6-thioinosine monophosphate (6-TIMP) (Figure 1, no. 9), 6-thioxanthosine monophosphate (6-TXMP) (Figure I, no. 10) and finally 6-thioguanine nucleotides (6-TGN) (Figure I, no. 11 and no. 12) which are thought to be the main active products of thioguanine derivatives. The mechanism of action of 6-TGN is still a matter of debate but its incorporation into DNA might trigger cell apoptosis and seems to be essential for cytotoxicity in leukaemia-derived cell lines. There is no polymorphism documented in the HGPRT gene but the Lesch Nyhan syndrome is characterized by a constitutive lack of activity of HGPRT. 6-TIMP (Figure I, no. 9) can also be metabolized by TPMT, producing methylated derivatives (MeTIMP) (Figure I, no. 16) that have a potential functional significance because they are inhibitors of phosphoribosylpyrophosphate amidotransferase (PPRPAT) (Figure I, no. 8), an enzyme involved in de novo purine synthesis. ${ }^{21}$ 6-TG (Figure I, no. 3) is metabolized mainly by HGPRT, giving rise to 6-TGN. It thus represents the most direct pathway to 6-TGN production.

\section{NEW TOOLS AND STRATEGIES PROPOSED TO OPTIMIZE TREATMENT WITH THIOGUANINE DERIVATIVES}

These tools and strategies are the result of a better knowledge of the metabolism of thioguanine derivatives (Figure I). They can be used at various times during treatment with these drugs, from the initiation of treatment to the decision to stop it.

\section{Initiation of treatment}

When treatment is initiated several points must be discussed: the indication for treatment, the starting dose, the route of administration, TPMT genotyping and the measurement of TPMT activity, and the choice between AZA and 6-MP. Very few data are available to discuss this last point. The initial choice between AZA or 6-MP is thought to be equivalent and the preferential use of one of these by reference teams has probably been linked to tradition with, for example, a more frequent use of 6-MP in the USA and AZA in Europe. It may still be a question of debate, however, because the nitro-imidazole metabolite (Figure I, no. 13) released from AZA may theoretically induce side-effects ${ }^{22}$ but could also have some immunomodulatory effects participating in the efficacy of the drug. ${ }^{16,23}$ Indication, starting dose and route of administration on the one hand and TPMT genotyping and measurement of activity on the other hand are potentially linked aspects.

\section{Indication, starting dose and route of administration of thioguanine derivatives}

AZA and 6-MP are largely considered to be second- or even third-line treatments in IBD. However, these molecules have been shown to be able to induce deep and prolonged mucosal healing ${ }^{24}$ and could therefore change the natural history of the disease. Some recent studies have tried to evaluate the benefit of an earlier induction of AZA or 6-MP treatment. In CD, the adjunction of 6-MP to steroids to treat active disease in children within 8 weeks of diagnosis was shown to be beneficial in a placebo-controlled study. ${ }^{25}$ Induced remission rates were similar in the two arms but co-treatment with 6-MP $1.5 \mathrm{mg} / \mathrm{kg} /$ day was associated with a lower relapse rate over 18 months ( 9 versus 47\%). Two remarks have to be made here: first, these impressive results need to be confirmed in adults, and second, there are no sufficient data today to support the systematic use of A2A or 6-MP in every patient with $\mathrm{CD}$. An important task would be to try and define at-risk populations who would benefit 
most from such early treatment. ${ }^{26}$ The use of AZA or 6-MP in active IBD has been hampered by slow action. An intravenous loading dose has been tried in order to reduce time to efficacy and also to extend the indication to severe active refractory disease. Preliminary results from small pilot studies were encouraging both in refractory $\mathrm{CD}^{27}$ and in severe refractory $\mathrm{UC} .^{28}$ However, a large controlled trial in $\mathrm{CD}$ has not confirmed the superiority of an intravenous loading dose of AZA over optimal oral dosage. ${ }^{29}$ In this last trial, however, the onset of action for oral AZA was quicker than previously admitted. Patients received $2 \mathrm{mg} / \mathrm{kg} / \mathrm{day}$ of AZA and response to treatment appeared after 2 weeks and reached a plateau after 4 weeks. Furthermore, steady-state concentration of active metabolites 6-TGN (Figure I, no. 11 and no. 12) was reached after 2-3 weeks. In comparison, a treatment starting with an intermediate dose of AZA, such as $1 \mathrm{mg} / \mathrm{kg}$, progressively increased to optimal dosage, may be associated with a slower onset of action. Such a progressive increase in dosage is used mainly for safety reasons but does not seem currently evidence-based.

Figure I: Metabolism of thioguanine derivatives (for details, see text). The drugs that can be used in IBD are azathioprine (AZA) (I), 6-mercaptopurine (6-MP) (2) and 6-thioguanine (6-TG) (3). Key enzymes involved in the metabolism of these drugs are: thiopurine methyl transferase (TPMT) (5), hypoxanthine guanine phosphoribosyl transferase (HGPRT) (6) and xanthine oxidase (XO) (7). Active metabolites are 6-thioguanime nucleotides (6-TGN) (11): 6-thioguanine mono-di-and triphosphate (6-TGMP, 6-TGDP, 6-TGTP) (12), which incorporate into DNA and RNA, and 6-methyl thioinosinic monophosphate (6-MeTIMP), which can interfere with de novo purine synthesis. Potentially toxic metabolites are 6-TGN (II), which can induce myelosuppression, and 6-methyl mercaptopurine (6-MMP) (15), which is probably involved in hepatotoxicity.

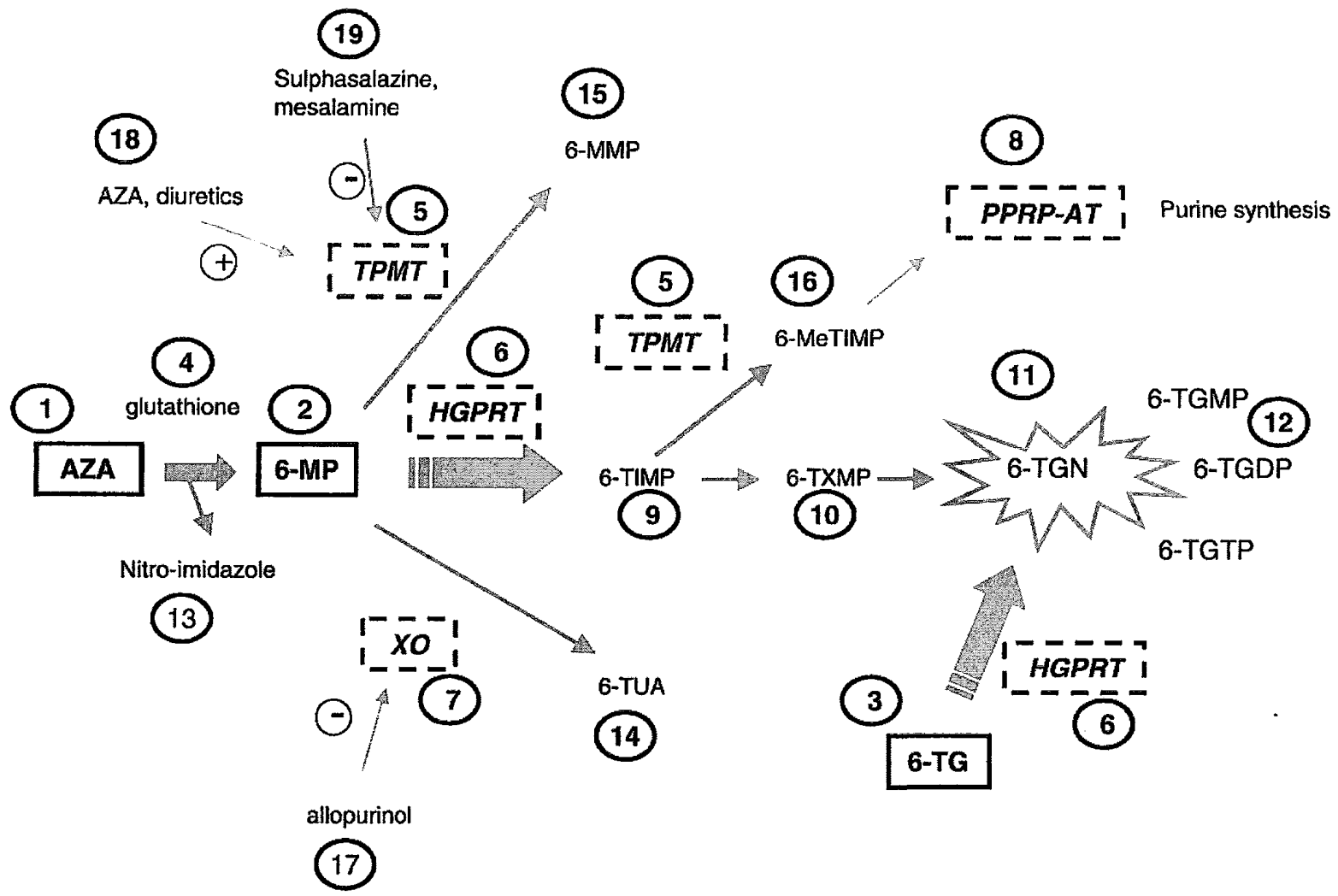

\section{TPMT genotyping and measurement of activity}

The rationale for TPMT genotyping or for TPMT activity measurement is, as mentioned above, that the activity of this enzyme is genetically determined and has a trimodal distribution, with intermediate- and, above all, lowmetabolizers being at risk of developing myelotoxicity due to preferential metabolism through HGPRT (Figure I, no. 6) and high production of 6-TGN metabolites (Figure I, no. 11 and no. 12). From a safety point of view it would seem wise to test before treatment in order to adapt the dosage of AZA or 6-MP from the start. Some remarks have to be made, however. First, when analysing the TPMT genotype in a large cohort of patients with severe myelotoxocity under AZA or 6-MP, it was found that only a minority of them had intermediate $(17 \%)$ or low-metabolizing $(10 \%)$ genotypes. ${ }^{30}$ This important study indicates that the majority of the bone marrow 
suppressions under AZA or 6-MP in IBD are not linked to TPMT genotype and that a normal, high metabolization profile in a patient does not supplant the need for the monitoring of white blood cells and platelets. Second, TPMT activity may vary over time, and measurement at the beginning of the treatment does not guarantee the level of activity over the following months or years. Third, at the doses used in IBD the risk of early severe myelosuppression essentially exists for TPMT-deficient homozygotes, who represent only $0.3 \%$ of the population. Hence, when analysing published data on myelotoxicity of AZA or 6-MP, it appears that even in the case of TPMT deficiency a severe myelosuppression does not usually occur within I week of treatment ${ }^{13,30}$ and can thus be disclosed adequately in the large majority of cases by weekly blood tests during the first month of treatment. Furthermore, a homozygote TPMT deficiency has probably no longer to be considered as an absolute contraindication for AZA or 6-MP treatment because these patients can be treated safely by adjusting the dosage to obtain the lowest effective levels of 6-TGN (Figure I, no. 11 and no. I2). ${ }^{31}$ This situation is actually close to the direct treatment with 6-TG (Figure I, no. 3), which has been advocated by some authors in cases of intolerance to AZA and 6-MP ${ }^{32}$ or high TPMT activity (Figure I, no. 5) which may be associated with relative resistance to AZA or 6-MP due to low 6-TGN production (Figure I, no. II and no. 12). ${ }^{33}$

\section{Early phase and chronic treatment}

Once treatment with thioguanine derivatives has been initiated there is a double goal to pursue: achieve efficacy and check for safety, mainly to avoid myelosuppression and hepatotoxicity. For safety, systematic blood cell count and liver function tests must be performed weekly during the first month and then monthly or at least every 2 months. As mentioned above, normal TPMT activity or genotyping does not supplant the need for regular surveillance of blood cell count. The strategy for achieving optimal efficacy is still controversial. Some authors advocate a standard dose with adjustment in non-responders, while others suggest a systematic target level of active metabolite, 6-TGN (Figure I, no. 11 and no. 12) or even the monitoring of more complex metabolites.

\section{Adjustment of dose}

Until a few years ago it was accepted that a patient non-responding to $1.5 \mathrm{mg} / \mathrm{kg} /$ day of 6-MPor $2-2.5 \mathrm{mg} / \mathrm{kg} / \mathrm{day}$ of AZA should be treated with other drugs. A first pilot study then suggested that an increase in AZA dosage beyond $2.5 \mathrm{mg} / \mathrm{kg}$ (up to $3-3.5 \mathrm{mg} / \mathrm{kg} / \mathrm{day}$ ) could induce a response in two-thirds of the patients. ${ }^{34}$ This was done under the control of a white blood cell count, provided there was not already a leukopenia. Instead of adjusting dosage according to white blood cell count, some authors suggest monitoring metabolites, mainly 6-TGN (Figure I, no. 11 and no. 12) and 6-MMP (Figure I, no. 15).

\section{Monitoring of AZA and 6-MP metabolites}

Several recent studies have analysed correlations between the levels of AZA/6-MP metabolites and the efficacy or toxicity of these drugs. A significant positive correlation was documented by some authors between the level of erythrocyte 6-TGN (Figure I, no. 11 and no. 12) and clinical response. ${ }^{19,35}$ A cut-off level of 235 or 250 $\mathrm{pmol} / 8 \times 10^{8}$ red blood cells was even proposed. However, this correlation was not confirmed by others, including the largest study on the topic. ${ }^{36,37}$ One explanation for this absence of correlation could be that the mechanism of action of AZA and 6-MP is more complex and depends also on other metabolites, including MeTIMP (Figure I, no. 16) that may inhibit de novo purine synthesis. ${ }^{21}$ Even in studies in which a positive correlation was found between erythrocyte 6-TGN and clinical response, a significant number of patients already responded while 6-TGN was below the cut-off level. Obviously these patients do not need an increased dosage of AZA or 6-MP. Even if a systematic target level of 6-TGN is not set during treatment, this metabolite may still be useful in non-responders, either to check for compliance or to increase the dosage under a control that is more specific than simply looking at the white blood cell count. ${ }^{36}$ A correlation was also documented between 6-MMP (Figure I, no. 15) levels and some side-effects, particularly hepatotoxicity. ${ }^{19,20}$ In some patients non-responding to AZA/6-MP and having particularly high TPMT (Figure I, no. 5) activity, an increased dose of AZA/6-MP resulted in minor clinical benefit, minor increase in 6-TGN but a significant increase in 6-MMP and dosedependent hepatotoxicity. ${ }^{20}$ It was therefore suggested that these patients, non-responding at standard doses and having high 6-MMP levels, should be treated with other drugs or even shifted to 6-TG (Figure I, no. 3).

\section{Intolerance and inefficacy}

Some problems of tolerance and efficacy cannot be solved by dose adjustment through monitoring of metabolites. These situations include allergic-type reactions and drug resistance despite dosage adjustment. In some of these situations, a shift from AZA to 6-MP or even 6-TG may be attempted, while some authors have 
tried to modulate TPMT activity.

Shift from AZA to 6-MP or 6-TG

Some side-effects with AZA may be due to the nitro-imidazole metabolite (Figure I, no. 13), released to give 6MP. Therefore, in some cases of intolerance to AZA, mainly digestive intolerance (apart from pancreatitis), arthralgia, malaise or rash, a treatment with 6-MP may be well tolerated in $50 \%$ of cases. ${ }^{38}$ As we have mentioned, hepatotoxicity may be linked to high 6-MMP levels (Figure I, no. 15). In this situation, a treatment with 6-TG has been suggested and seems effective and well tolerated. ${ }^{32} \mathrm{~A}$ shift to 6-TG has also been proposed when AZA or 6-MP are ineffective due to low levels of 6-TGN (Figure I, no. 11 and no. 12) being achieved. ${ }^{33}$ However, the data on safety and efficacy of 6-TG in these situations are still preliminary. It must be mentioned that the levels of 6-TGN (Figure I, no. 11 and no. 12) achieved under 6-TG treatment are much higher than with AZA and 6-MP, and that some concerns exist due to reports of an up to ninefold increased risk of secondary malignancy in TPMT heterozygous children treated with thiopurines for remission maintenance in acute lymphoblastic leukaemia. ${ }^{39}$

\section{Modulation of TPMT activity}

A relative decrease in TPMT activity (Figure I, no. 5) may favour the HGPRT (Figure I, no. 6) pathway and therefore increase the production of active metabolites (6-TGN, Figure I, no. 11 and no. 12). Sulphasalazine and mesalamine may inhibit TPMT activity (Figure I, no. 19) ${ }^{18}$ Sulphasalazine treatment was introduced in a small number of patients, non-responding to 6-MP, who had high levels of 6-MMP (Figure I, no. 15) and a relatively low level of 6-TGN (Figure I, no. 11 and no. 12) ${ }^{20}$; however, this was not useful in terms of clinical efficacy or even in the profile of metabolites. Other ways of TPMT modulation should be explored.

\section{When to stop AZA/6-MP treatment}

Stopping AZA/6-MP treatment is associated with a high relapse rate. A retrospective study, however, showed that the risk of relapse was lower in patients who had been in remission on AZA/6-MP for a longer period of time. The risk seemed similar in patients stopping or carrying on with the drug when it was stopped beyond 4 years of induced remission. ${ }^{4}$ This was recently re-assessed in a prospective placebo-controlled study. ${ }^{40}$ Patients were followed up for 18 months after randomization in the AZA or placebo group. The relapse rate was $21 \%$ in the placebo group versus $8 \%$ in the AZA group, and the statistical analysis showed that these results were not equivalent. These results do not confirm previous retrospective data and indicate that the patient still benefits from AZA treatment even after 4 years of remission. Interestingly, a persistent elevated level of C-reactive protein under AZA treatment was associated with a higher risk of relapse after stopping treatment.

\section{SUMMARY}

Thioguanine derivatives, mainly AZA and 6-MP, are major drugs in the treatment of chronic active, steroiddependent and infliximab-treated patients. They are effective in two-thirds of the patients and are well tolerated over the long term. Over the last 10 years, progress has been made in understanding the metabolism of thioguanine derivatives and its implication in clinical practice. New tools and strategies are proposed to try and improve both the efficacy and safety of these drugs. TPMT genotyping or activity measurement may be helpful before starting the treatment to disclose patients at risk of severe myelosuppression. This has not been prospectively validated, however, and these procedures do not supplant the need for regular blood test surveillance during the treatment. Starting the treatment directly with the optimal dosage $(2-2.5 \mathrm{mg} / \mathrm{kg} / \mathrm{day}$ for AZA and $1-1.5 \mathrm{mg} / \mathrm{kg} /$ day for 6-MP) probably accelerates the onset of action. No evidence exists to recommend a systematic monitoring of AZA/6-MP metabolites, mainly 6-TGN and 6-MMP, but these measurements can help in non-responders to check for compliance and decide on increases in dosage or changes in immunosuppressor. In some cases of intolerance to AZA, 6-MP can be tried and, if intolerance exists to both of these drugs, 6-TG may be helpful. The risk of relapse when the treatment is stopped decreases with the duration of induced remission, but patients still benefit from the drug beyond 4 years of treatment.

\section{Practice points}

- the usefulness of TPMT genotyping and/or activity measurement before starting treatment remains controversial

- starting treatment with the optimal theoretical dose of AZA (2-2.5 mg/kg) or 6-MP (1-1.5 mg/kg) probably accelerates the onset of action 
- in patients non-responding to a standard dose of AZA/6-MP, dosage can be increased under the control of the white blood cell count or erythrocyte 6-TGN concentration

- counts of white cells and platelets, as well as liver function tests, must be performed regularly in every patient treated with AZA/6-MP

- some patients intolerant to AZA may tolerate 6-MP, and the majority of the patients intolerant to 6-MP can tolerate 6-TG

- treatment with AZA/6-MP in IBD should be carried on beyond 4 years as long as it is well tolerated and there is no good reason to stop

\section{Research agenda}

- the interest in, and cost-effectiveness of, a systematic TPMT evaluation before treatment must be prospectively evaluated

- a prospective comparison of AZA and 6-MP tolerance and efficacy would be useful

- the interest in, and need for, an intravenous loading dose with AZA has still to be further evaluated

- interest in, and cost-effectiveness of, systematic monitoring with 6-TGN and 6-MMP to improve AZA/6-MP treatment must be prospectively evaluated

- the usefulness of 6-TGN measurement in patients in whom AZA/6-MP dosage is increased beyond standard doses must be prospectively compared to the simple white blood cell count

- safety, efficacy and optimal dosage of 6-TG in IBD must be evaluated in controlled trials

- attempts at modulating TPMT activity may be relevant to IBD treatment and must be evaluated

\section{REFERENCES}

* 1. Pearson DC. May GR, Fick GH \& Sutherland LR. Azathioprine and 6-mercaptopurine in Crohn's disease. A meta-analysis. Annals of Internal Medicine 1995; 123: 132-142.

2. Present DH, Korelitz B1, Wisch N et al. Treatment of Crohn's with 6-mercaptopurine: a long-term, randomized, double-blind study. New England Journal of Medicine 1980; 302: 981-987.

3. Ewe K, Press AG, Singe CC et al. Azathioprine combined with prednisolone or monotherapy with prednisolone in active Crohn's disease Gastroenterology 1993; 105: 367-372.

4. Bouhnik Y, Lémann M, Mary JY et al. Long-term follow up of patients with Crohn's disease treated with azathioprine or 6mercaptopurine. Lancet 1996; 347: 215-219.

5. Hawthorne AB, Logan RFA, Hawkey CJ et al. Randomised controlled trial of azathioprine withdrawal in ulcerative colitis. British Medical Journal 1992; 305: 20-22.

6. Vermeire S, Louis E, Carbonez A et al. Logistic regression of clinical parameters influencing response to infliximab. Gastroenterology 2001; 120: 3149

7. Farrell RJ, Alsahli M, Falchuk KR et al. Human anti-chimeric antibody levels correlate with lack of response and infusion reactions following infliximab therapy. Gastroenterology 2001; 120: 364.

8. Fraser AG, Orchard TR \& Jewell DP. The efficacy of azathioprine for the treatment of inflammatory bowel disease: a 30 year review. Gut 2002; 50: 485-489

9. Rosenberg JL, Wall A), Levin B et al. A controlled trial of azathioprine, in the management of chronic ulcerative colitis. Gastroenterology 1975; 69: 96-99.

10. Kirk AP \& Lennard-Jones JE. Controlled trial of azathioprine in chronic ulcerative colitis. British Medical Journal 1982; 284: 12911292.

11. Georges J, Present DH, Pou R et al. The long-term outcome of ulcerative colitis treated with 6-mercaptopurine. American Journal of Gastroenterology 1996; 91: 1711-1714.

*12. Present DH, Meltzer SJ, Krumholtz MP et al. 6-mercaptopurine in the management of inflammatory bowel disease: short- and longterm toxicity. Annals of Internal Medicine 1989; 111: 641-649.

13. Connel WR, Kamm MA, Ritchie JK \& Lennard-Jones JE. Bone marrow toxicity caused by azathioprine in inflammatory bowel disease: 27 years of experience. Gut 1993; 34: 1081-1085.

*14. Connel WR, Kamm MA, Dickson M et al. Long-term neoplasia risk after azathioprine treatment in inflammatory bowel disease. Lancet 1994; 343: 1249-1251. 
Published in: Best Practice (2003), vol.17, pp.37-46

Status: Postprint (Author's version)

15. Blondel-Kucharski F, Chircop C, Marquis P et al. and the GETAID. Health-related quality of life in Crohn's disease: a prospective longitudinal study in 231 patients. American Journal of Gastroenterology 2001;96:2915-2920.

16. Crawford DJ, Maddocks J, Jones DN \& Szalowski P. Rational for design of novel immunosuppressive drugs: analogues of azathioprine lacking the 6-mercaptopurine substituent retain or have enhanced immunosuppressive effects. Journal of Medical Chemistry 1996; $39: 63-68$.

17. Weinshilboum RM \& Sladek SL. Mercaptopurine pharmacogenetics; monogenic inheritance of erythrocyte thiopurine methyltransferase activity. American Journal of Human Genetics 1980; 32: 651-652.

18. Szumlanski CL \& Weinshilboum RM. Sulphasalazine inhibition of thiopurine methyltransferase: possible mechanism for interaction with 6-mercaptopurine and azathioprine. British Journal of Clinical Pharmacology 1995; 39: 456-459.

19. Dubinsky MC, Lamothe S, Yang HY et al. Pharmacogenomics and metabolite measurement for 6-mercaptopurine therapy in inflammatory bowel disease. Gastroenterology 2000; 118: 705-713.

*20. Dubinsky MC, Yang H, Hassard PV et al. 6-MP metabolite profiles provide a biochemical explanation for 6-MP resistance in patients with inflammatory bowel disease. Gastroenterology 2002; 122: 904-915.

21. Vogt M, Stet EH, De Abreu RA et al. The importance of methylthio-IMP for methylmeraptopurine ribonucleoside (Me-MPR) cytotoxicity in Molt F4 human malignant T-lymphoblasts. Biochimica et Biophysica Acta 1993; 1181: 189-194.

22. McGovern DP, Travis SP, Duley J et al. Azathioprine intolerance in patients with IBD may be imidazole-related and is independent of TPMT activity. Gastroenterology 2002; 122: 838-839.

23. Louis E, Aboul Nasr El Yafi F \& Belaiche J. High dose of azathioprine but not 6-mercaptopurine inhibit the production of proinflammatory cytokines in inflammatory bowel disease. Gastroenterology 2000; 118: 4204.

24. D'Haens G, Geboes K, Ponette E et al. Healing of severe recurrent ileitis with azathioprine therapy in patients with Crohn's disease. Gastroenterology 1997; 112: 1475-1481.

*25. Markowitz J, Grancher K, Kohn N et al and the pediatric 6-MP collaborative group. A multicenter trial of 6-Mercaptopurine and prednisone in children with newly diagnosed Crohn's disease. Gastroenterology 2000; 119: 895-902.

26. Seidman EG. 6-mercaptopurine in maintaining remission in Crohn's disease: an old friend becomes a new hero. Gastroenterology 2000; 119: $1158-1164$.

27. Sandborn WJ, van Os EC, Zins BJ et al. An intravenous loading dose of azathioprine decreases the time to response in patients with Crohn's disease. Gastroenterology 1995; 109: 1808-1817.

28. Mahadevan U, Tremaine WJ, Johnson T et al. Intravenous azathioprine in severe ulcerative colitis: a pilot study. American Journal of Gastroentrology 2000; 95: 3463-3468.

*29. Sandborn WJ, Tremaine WJ, Wolf DC et ai for the north American azathioprine study group. Lack of effect of intravenous administration on time to respond to azathioprine for steroid-treated Crohn's disease. Gastroenterology 1999; 117: 527-535.

*30. Colombel JF, Ferrari N, Debuysere H et al. Genotypic analysis of thiopurine s-methyltransferase in patients with Crohn's disease and severe myelosuppression during azathioprine therapy. Gastroenterology 2000; 118: 1025-1030.

31. Kaskas B, Louis E, Hindorf U et al. TPMT-deficient patients with inflammatory bowel disease can be treated safely with azathioprine after dose adjustment. Gut 2002: (in press).

32. Derijks LJ, De Jong DJ, Gilissen LP et al. 6-thioguanine in azathioprine and 6-mercaptopurine intolerant patients with inflammatory bowel disease. British Journal of Clinical Pharmacology 2001; 53: 546P-547P.

33. Dubinsky MC, Hassard PV, Seidman EG et al. An open-label pilot study using thioguanine as a therapeutic alternative in Crohn's disease patients resistant to 6-mercaptopurine therapy. Inflammatory Bowel Disease 2001; 7: 181-189,

34. Barbe L, Marteau P, Lémann M et al. Dose raising of azathioprine beyond $2.5 \mathrm{mg} / \mathrm{kg} / \mathrm{d}$ in Crohn's disease who fail to improve with a standard dose. Gastroenterology 1998; 114: G3794.

*35. Cuffari C, Hunt S \& Bayless T. Utilisation of erythrocyte 6-thioguanine metabolite levels to optimize azathioprine therapy in patients with inflammatory bowel disease. Gut 2001; 48: 642-646.

36. Balaiche J, Desager JP, Horsmans Y \& Louis E. Therapeutic drug monitoring of azathioprine and 6-mercaptopurine metabolites in Crohn's disease. Scandinavian Journal of Gastroenterology 2001; 36:71-76.

*37. Lowry PW, Franklin CL, Weaver AL et al. Measurement of thiopurine methyltransferase activity and azathioprine metabolites in patients with inflammatory bowel disease. Gut 2001; 49: 665-670. 
Published in: Best Practice (2003), vol.17, pp.37-46

Status: Postprint (Author's version)

38. Lémann M, Bouhnik Y, Kmieciak-le-Corguille M et al. Treatment of Crohn's disease with 6-mercaptopurine in patients intolerant to azathioprine. Gastroenterology 1997; 112: A 1025.

39. Thomsen JB, Schroder H, Kristinsson J et al. Possible carcinogenic effect of 6-mercaptopurine on bone marrow stem cells. Relation to thiopurine metabolism. Cancer 1999; 86: 1080-1086.

*40. Lémann M, Bouhnik Y, Colombel JF et al. Randomized, double-blind, placebo-controlled, multicenter, azathioprine (AZA) withdrawal trial in Crohn's disease. Gastroenterology 2002; 122: 174. 